\title{
FRANK-CONDON PRINCIPLE \\ AND ADJUSTMENT OF OPTICAL WAVEGUIDES WITH NONHOMOGENEOUS REFRACTIVE INDICES
}

\author{
Vladimir I. Man'ko', Leonid D. Mikheev", and Alexandr Sergeevich² \\ ${ }^{1}$ P. N. Lebedev Physical Institute, Russian Academy of Sciences \\ Leninskii Prospect 53, Moscow 119991, Russia \\ ${ }^{2}$ School of Physics, The University of Sydney \\ New South Wales 2006, Australia \\ *Corresponding author, e-mail: a.sergeevich@physics.usyd.edu.au \\ e-mails:manko@sci.lebedev.ru mikheev@sci.lebedev.ru
}

\begin{abstract}
The adjustment of two different selfocs is considered using both exact formulas for the modeconnection coefficients expressed in terms of Hermite polynomials of several variables and a qualitative approach based on the Frank-Condon principle. Several examples of the refractive-index dependence are studied and illustrative plots for these examples are presented. The connection with the tomographic approach to quantum states of a two-dimensional oscillator and the Frank-Condon factors is established.
\end{abstract}

Keywords: Frank-Condon principle, entanglement, optical waveguide, molecular spectra, Hermite polynomials.

\section{Introduction}

It is known that radiation beams propagating in optical waveguides can be described by Schrödingerlike equations $[1-6]$; this has been studied in [7-20]. In this equation, the longitudinal coordinate $z$ plays the role of time and the radiation wavelength plays the role of the Planck's constant. The refractive-index profile is an analog of the potential energy in the quantum Schrödinger equation. The electromagnetic radiation modes in the optical waveguide are analogs of the wave functions of a quantum system. This quantumlike picture of the field propagating in the waveguide was widely used to study the energy distribution among the modes of successive two (or several) waveguides with different refractive index profiles, if the distribution in the first waveguide is known. This problem 
is equivalent to the quantum-mechanical problem of the vibronic structure of electronic lines in the absorption and emission spectra of polyatomic molecules. In the case of the harmonic potential, the structure is determined in terms of the Frank-Condon factor expressed through Hermite polynomials of several variables [21-23] (see, also [24-30]). Recently the technique of femtosecond pulses was developed and applied in different domains of ultrapower laser physics [31, 32] where the problem of waveguiding such kinds of radiation also arises [33, 34].

Our aim here is to consider analogs of the quantum problems of the molecular spectra discussed in [27-30] to transfer the results of these investigations into the domain of femtosecond-pulse laser physics. The other aim is to connect the classical problem of optical waveguides with the quantum problem of entanglement [35, 36]. The point is that for states with two degrees of freedom the notion of entanglement corresponds to the degree of correlation of observables related to these different degrees of freedom. In the waveguide picture, an analog of the entanglement is related to the structure of the modes propagating in the waveguides. When the initial separable two-mode field in the first waveguide is propagating into the second waveguide with a different profile of the refractive index, the entanglement appears in the arising modes in the second waveguide. The entanglement can be considered and related to the energy distribution among the field modes in the second waveguide. Here we discuss this relation and consider the possibility to find connections with different criteria of the entanglement [37-41].

The paper is organized as follows.

In Sec. 2, we review the results related to the modes in planar waveguides with quadratic refractive index. In Sec. 3 the two-mode problem in selfoc-like waveguides is considered, and an analog of the two-mode squeezed-light wave function is used for studying the Frank-Condon factor. The entanglement analogs of the waveguide modes are discussed in Sec. 4. The conclusion and perspectives are presented in Sec. 5.

\section{Planar Waveguides}

In this section, we consider the light-beam propagation from one planar waveguide with a quadratic refractive index to another waveguide with the propagation direction along the $z$ coordinate. The refractive index, being constant along the $y$ and $z$ axes, is described by a symmetric potential-energy curve of a one-electron atom as follows:

$$
n(x)=k x^{2} \leftrightarrow U(x)=\frac{\omega^{2} x^{2}}{2} .
$$

The second waveguide has a different dependence of the refractive index, and its axis is shifted from the axis of the first waveguide by $d$

$$
n^{\prime}(x)=k^{\prime}(x-d)^{2} \leftrightarrow U(x)=\frac{\omega^{\prime 2}(x-d)^{2}}{2},
$$

which is equivalent to shifting and stretching or shrinking of the potential-energy curve. Here we take $m=m^{\prime}=1$. 

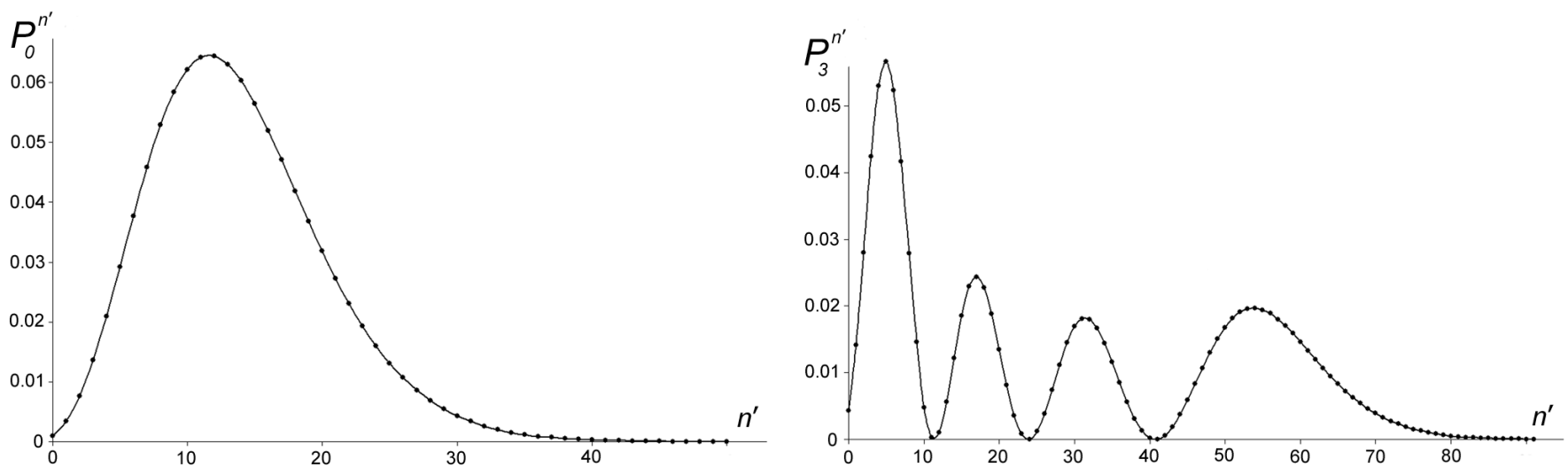

Fig. 1. The plot of $P_{0}^{n^{\prime}}$ for $\omega^{\prime} / \omega=3$ and $\omega d^{2}=9$.

Fig. 2. The plot of $P_{3}^{n^{\prime}}$ for $\omega^{\prime} / \omega=3$ and $\omega d^{2}=16$.

The most probable energy level to which the electron jumps can be found in view of the FrankCondon principle. Taking into consideration that the energy levels in a parabolic potential are distributed as $E_{n}=\hbar \omega(n+1 / 2)$, the final state can be easily found.

We consider a one-electron atom. The nuclear Hamiltonian in the Born-Oppenheimer approximation reads

$$
\mathcal{H}=\frac{1}{2} \hbar \omega\left\{a, a^{\dagger}\right\}
$$

where $\omega$ is the frequency corresponding to the coordinate $q, a$ and $a^{\dagger}$ are the creation and annihilation operators, respectively. The wave function of electron in the atom with Hamiltonian (3) is described by

$$
\psi(x, n, \omega)=\left(\pi^{1 / 2} 2^{n} n ! l(\omega)\right)^{-1 / 2} \exp \left(-\frac{x^{2}}{2 l^{2}(\omega)}\right) H_{n}\left(\frac{x}{l(\omega)}\right),
$$

where $l(\omega)=\sqrt{\hbar / \omega}, n$ is a vibrational quantum number, and $H_{n}(\xi)$ is the $n$th Hermite polynomial. Shifting the center of the potential from $x=0$ to $x=d$ and changing $\omega \rightarrow \omega^{\prime}$ and $n \rightarrow n^{\prime}$ provides the wave function

$$
\psi\left(x, n^{\prime}, \omega^{\prime}, d\right)=\left(\pi^{1 / 2} 2^{n^{\prime}} n^{\prime} ! l\left(\omega^{\prime}\right)\right)^{-1 / 2} \exp \left(-\frac{(x-d)^{2}}{2 l^{2}\left(\omega^{\prime}\right)}\right) H_{n^{\prime}}\left(\frac{x-d}{l\left(\omega^{\prime}\right)}\right) .
$$

The overlap integral

$$
\left\langle n \mid n^{\prime}\right\rangle=\int \psi(x, n, \omega, 0) \psi\left(x, n^{\prime}, \omega^{\prime}, d\right) \mathbf{d} x
$$

describes the transition probability amplitude for

$$
\psi(x, n, \omega, 0) \rightarrow \psi\left(x, n^{\prime}, \omega^{\prime}, d\right) .
$$

The integral (6) can be expressed through the Hermite polynomial of two variables

$$
\left\langle n \mid n^{\prime}\right\rangle=\left(2^{n+n^{\prime}} n ! n^{\prime} !\right)^{-1 / 2} \sqrt{\frac{2 l(\omega) l\left(\omega^{\prime}\right)}{l(\omega)^{2}+l\left(\omega^{\prime}\right)^{2}}} \exp \left(-\frac{d^{2}}{2\left(l(\omega)^{2}+l\left(\omega^{\prime}\right)^{2}\right)}\right) H_{n n^{\prime}}^{\{R\}}\left(y_{1}, y_{2}\right) .
$$


The arguments of the Hermite polynomial read

$$
\begin{aligned}
& R=\frac{2}{l(\omega)^{2}+l\left(\omega^{\prime}\right)^{2}}\left(\begin{array}{cc}
l(\omega)^{2}-l\left(\omega^{\prime}\right)^{2} & -2 l(\omega) l\left(\omega^{\prime}\right) \\
-2 l(\omega) l\left(\omega^{\prime}\right) & -l(\omega)^{2}+l\left(\omega^{\prime}\right)^{2}
\end{array}\right) \\
& \left(\begin{array}{l}
y_{1} \\
y_{2}
\end{array}\right)=-\frac{d l(\omega)}{l(\omega)^{2}+l\left(\omega^{\prime}\right)^{2}}\left(\begin{array}{c}
1 \\
-l\left(\omega^{\prime}\right) / l(\omega)
\end{array}\right)
\end{aligned}
$$

The transition probability from the state $|n\rangle$ to the state $\left|n^{\prime}\right\rangle$ is equal to

$$
P_{n}^{n^{\prime}}=\left(\left\langle n \mid n^{\prime}\right\rangle\right)^{2}
$$

To illustrate the probability distribution, we present in Fig. 1 a plot of the function $P_{n}^{n^{\prime}}$ for the initial level $n=0$, potential stretching $\omega^{\prime} / \omega=3$ and shift $\omega d^{2}=9$. The maximum probability is observed for the thirteenth level. This result is in agreement with the number calculated directly from the Frank-Condon principle. The plot for the transition from $n=3$ with $\omega^{\prime} / \omega=3$ and $\omega d^{2}=16$ is shown in Fig. 2. In this case, the most probable final state is $|5\rangle$.

\section{Elliptic Waveguides}

Now we extend our consideration to the case of elliptical waveguides with quadratic refractive index, which corresponds to a one-electron atom with the 2D parabolic potential,

$$
n(x, y)=k_{x} x^{2}+k_{y} y^{2} \leftrightarrow U(x, y)=\frac{\omega_{x} x^{2}+\omega_{y} y^{2}}{2}
$$

In complete analogy with the previous section, the shifted and deformed potential reads

$$
U^{\prime}(x, y)=\frac{\omega_{x}^{\prime}\left(x-d_{x}\right)^{2}+\omega_{y}^{\prime}\left(y-d_{y}\right)^{2}}{2} .
$$

The unshifted wave function of the electron in potential (11) is

$$
\psi\left(x, y, n_{x}, n_{y}, \omega_{x}, \omega_{y}, 0,0\right)=\frac{\exp \left[-\frac{1}{2}\left(\frac{x^{2}}{l^{2}\left(\omega_{x}\right)}+\frac{y^{2}}{l^{2}\left(\omega_{y}\right)}\right)\right]}{\left(\pi 2^{n_{x}+n_{y}} n_{x} ! n_{y} ! l\left(\omega_{x}\right) l\left(\omega_{y}\right)\right)^{1 / 2}} H_{n_{x}}\left(\frac{x}{l\left(\omega_{x}\right)}\right) H_{n_{y}}\left(\frac{y}{l\left(\omega_{y}\right)}\right) .
$$

In the same way, we can find the probability distribution in the $2 \mathrm{D}$ case for the transition

$$
\psi\left(x, y, n_{x}, n_{y}, \omega_{x}, \omega_{y}, 0,0\right) \rightarrow \psi\left(x, y, n_{x}^{\prime}, n_{y}^{\prime}, \omega_{x}^{\prime}, \omega_{y}^{\prime}, d_{x}, d_{y}\right)
$$

by calculating the overlap integral

$$
\left\langle n \mid n^{\prime}\right\rangle=\int \psi\left(x, y, n_{x}, n_{y}, \omega_{x}, \omega_{y}, 0,0\right) \psi\left(x, y, n_{x}^{\prime}, n_{y}^{\prime}, \omega_{x}^{\prime}, \omega_{y}^{\prime}, d_{x}, d_{y}\right) \mathbf{d} x \mathbf{d} y .
$$

Figure 3 presents the distribution for the transition from the ground state for $\omega_{x}^{\prime} / \omega_{x}=2, \omega_{y}^{\prime} / \omega_{y}=$ $3, \omega_{x} d_{x}^{2}=9$, and $\omega_{y} d_{y}^{2}=16$. The maximum of this function is observed for the final state $|23,8\rangle$. 


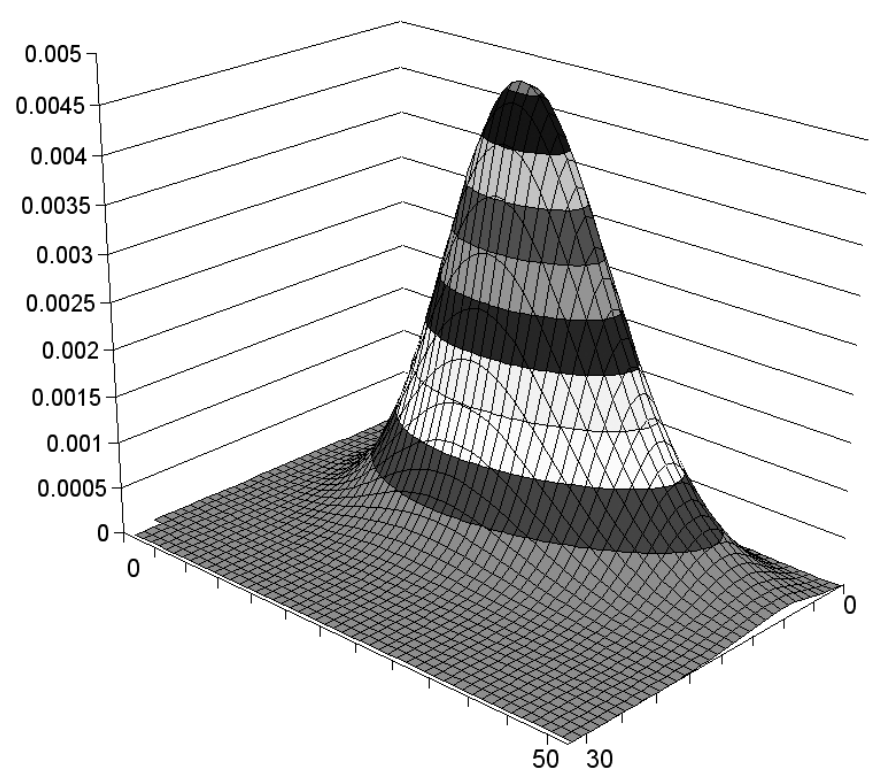

Fig. 3. Plot of $P_{0,0}^{n_{x}^{\prime}, n_{y}^{\prime}}$ for $\omega_{x}^{\prime} / \omega_{x}=2, \omega_{y}^{\prime} / \omega_{y}=3$, $\omega_{x} d_{x}^{2}=9$, and $\omega_{y} d_{y}^{2}=16$.

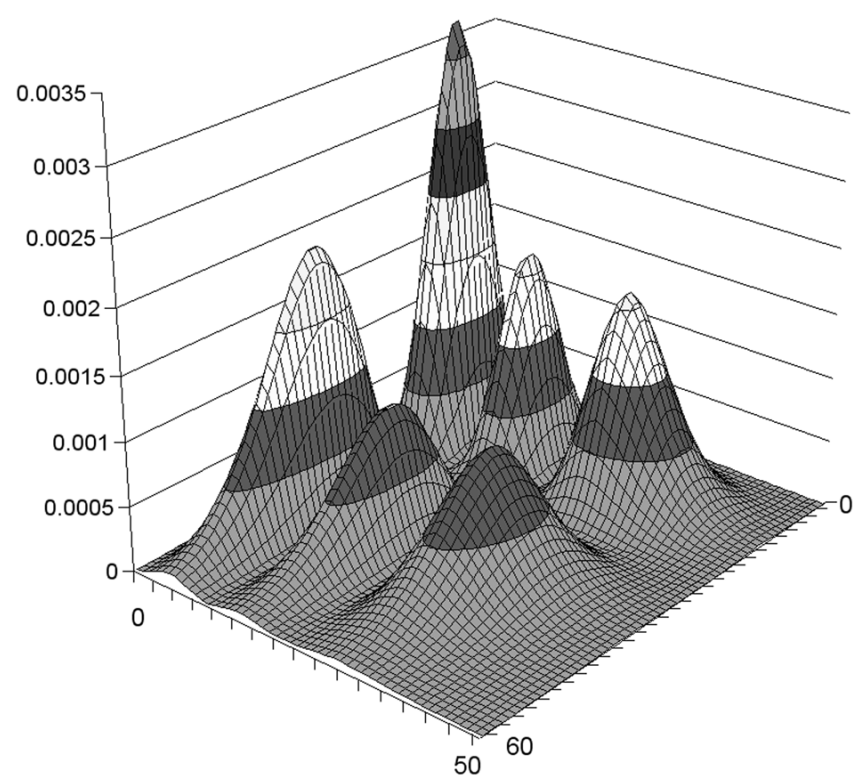

Fig. 4. Plot of $P_{2,1}^{n_{x}^{\prime}, n_{y}^{\prime}}$ for $\omega_{x}^{\prime} / \omega_{x}=2, \omega_{y}^{\prime} / \omega_{y}=3$, $\omega_{x} d_{x}^{2}=16$, and $\omega_{y} d_{y}^{2}=16$.

In Fig. 4, a 3D plot of the transition probability for the initial state $|2,1\rangle$ and $\frac{\omega_{x}^{\prime}}{\omega_{x}}=2, \frac{\omega_{y}^{\prime}}{\omega_{y}}=3$, and $\omega_{x} d_{x}^{2}=\omega_{y} d_{y}^{2}=16$ is presented. The state $|8,4\rangle$ is the most probable final state.

The pictures are obviously very similar to the planar case. The transition probability distribution for the initial state $|0,0\rangle$ has the Gaussian form. For the transitions from the excited state, one also can observe a multi-maxima surface.

Since there is no $x y$ component in potential (12), the wave created as a result of transition does not contain the entanglement term. To obtain a state analogous to the entangled state, one should consider the potential with such a term, i.e.,

$$
U(x, y)=\frac{\omega_{x} x^{2}+\omega_{y} y^{2}+\gamma x y}{2} .
$$

After the transition, the wave function has the form

$$
\psi(x, y)=\frac{\exp \left[-\frac{1}{2}\left(\frac{x^{2}}{l^{2}\left(\omega_{x}\right)}+\frac{y^{2}}{l^{2}\left(\omega_{y}\right)}+\gamma x y\right)\right]}{\left(\pi 2^{n_{x}+n_{y}} n_{x} ! n_{y} ! l\left(\omega_{x}\right) l\left(\omega_{y}\right)\right)^{1 / 2}} H_{n_{x}}(a x+b y) H_{n_{y}}(c x+d y) .
$$

As a result, the probability distribution is changed slightly in this case, so we present just plots for potential 12 with $\gamma=0$, keeping in mind that the real plots are very close to these ones.

\section{Conclusions}

To conclude, we point out the main results of the paper. 
We reviewed the approach for describing the light propagation in optical waveguides using the Fock-Leontovich approximation. In this approximation, the equation describing the light intensity and phase is identical to the Schrödinger equation. In view of this, following [24] we applied an analog of the Frank-Condon principle to express the energy distribution among the modes in the optical waveguide by the Frank-Condon factors. For harmonic potential, these factors are given in the explicit form in terms of special functions associated with Hermite polynomials. We made an analysis of some concrete examples and investigated the energy-distribution function for the light modes in the optical waveguide. The distribution functions were shown to have maxima corresponding to the Frank-Condon principle.

\section{Acknowledgments}

V.I.M. acknowledges the support of the Russian Foundation for Basic Research under Projects Nos. 07-02-00598 and 08-02-90300.

\section{References}

1. M. A. Leontovich, Izv. Akad. Nauk SSSR, 8, 16 (1944).

2. M. A. Leontovich and V. A. Fock, Zh. Éksp. Teor. Fiz., 16, 557 (1946).

3. J. Plebański, "Classical properties of oscillator wave packets," Bull. Acad. Pol. Sci., 2, 213 (1954).

4. D. Gloge and D. Marcuse, J. Opt. Soc. Am., 59, 1629 (1969).

5. D. Marcuse, Light Transmission Optics, Van Nostrand, New York (1972).

6. J. A. Arnaud, Beam and Fiber Optics, Academic, New York (1976).

7. M. A. Man'ko and G. T. Mikaelyan, Kvantov. Élektron., 13, 1506 (1986) [Sov. J. Quantum Electron., 16, 201 (1986)].

8. M. A. Man'ko and G. T. Mikaelyan, The Nonlinear Optics of Semiconductor Lasers, Proceedings of the P.N. Lebedev Physical Institute, Nauka, Moscow (1986), Vol. 166, p. 126 [Nova Science, Commack, New York (1987), Vol. 166, p. 170].

9. M. A. Man'ko, "Some aspects of nonlinear optics of semiconductor lasers," in: M. Bertolotti (ed.), ECOOSA-90 Quantum Optics, IOP Publishers, Bristol (1991), p. 247.

10. R. Fedele and P. K. Schukla (eds.) Quantumlike Models and Coherent Effects, World Scientific, Singapore (1995).

11. S. De Martino, S. De Nicola, S. De Siena, R. Fedele, and G. Miele (eds.), Quantumlike Description and Macroscopic Coherent Phenomena, World Scientific, Singapore (1997). 
12. R. Fedele and V. I. Man'ko, Phys. Rev. E, 58, 992 (1998).

13. R. Fedele, M. A. Man'ko, and V. I. Man'ko, J. Russ. Laser Res., 21, 1 (2000).

14. R. Fedele, M. A. Man'ko, and V. I. Man'ko, J. Opt. Soc. Am. A, 17, 2506 (2000).

15. M. A. Man'ko, J. Russ. Laser Res., 22, 48 (2001).

16. M. A. Man'ko, V. I. Man'ko, and R. V. Mendes, Phys. Lett. A, 288, 132 (2001).

17. R. Fedele and M. A. Man'ko, Eur. Phys. J. D, 27, 263 (2003).

18. S. Chávez-Cerda, J. R. Moya-Cessa, and H. M. Moya-Cessa, J. Opt. Soc. Am. B, 24, 404 (2007).

19. M. A. Man'ko, Eur. Phys. J. Spec. Top., 160, 269 (2008).

20. M. A. Man'ko, J. Phys. Conf. Ser., 99, 012012 (2008).

21. E. V. Doktorov, I. A. Malkin, and V. I. Man’ko, J. Mol. Spectrosc., 56, 1 (1975).

22. E. V. Doktorov, I. A. Malkin, and V. I. Man'ko, J. Mol. Spectrosc., 64, 302 (1977).

23. I. A. Malkin and V. I. Man'ko, Dynamical Symmetries and Coherent States of Quantum Systems [in Russian], Nauka, Moscow (1979).

24. M. A. Man'ko and G. T. Mikaelyan, Sov. J. Quantum Electron., 16, 201 (1986).

25. O. Castaños, R. López-Peña, and R. Lemus, J. Mol. Spectrosc., 241, 51 (2007).

26. F. Santaro, R. Improta, A. Lami, et al., J. Chem. Phys., 126, 084509 (2007).

27. A. Toniolo and M. Persico, J. Comput. Chem., 22, 968 (2001).

28. H. Kikuchi, M. Kubo, N. Watanabe, and H. Suzuki, J. Chem. Phys., 119, 729 (2003).

29. P. T. Ruhoff, Chem. Phys., 186, 355 (1994).

30. P. T. Ruhoff and M. A. Ratner, Int. J. Quantum Chem., 77, 383 (2000).

31. L. D. Mikheev, Laser Part. Beams, 10, 473 (1992).

32. V. I. Tcheremiskine, M. L. Sentis, and L. D. Mikheev, Appl. Phys. Lett., 81, 403 (2002).

33. S. Jackel, R. Burris, J. Grun, et al., Opt. Lett., 10, 1086 (1995).

34. F. Gerome, K. Cook, A. K. George, et al., Opt. Express, 12, 835 (2004).

35. E. Schrödinger, Proc. Cambridge Philos. Soc., 31, 555 (1935). 
36. A. Einstein, B. Podolsky, and N. Rosen, Phys. Rev., 47, 777 (1935).

37. V. I. Man'ko and A. A. Sergeevich, J. Russ. Laser Res., 28, 516 (2007).

38. R. Simon, Phys. Rev. Lett., 84, 2726 (2000).

39. A. S. Chirkin and M. Yu. Saigin, Acta Phys. Hung. B, 26/1-2, 63 (2006).

40. M. Yu. Saigin and A. S. Chirkin, Mod. Probl. Stat. Phys., 5, 169 (2006).

41. A. S. Chirkin and M. Yu. Saigin, J. Russ. Laser Res., 28, 505 (2007). 